\title{
FIXED POINT FREE ACTIONS OF GROUPS OF EXPONENT 5
}

\author{
ENRICO JABARA
}

(Received 8 April 2002; revised 11 April 2003)

Communicated by R. B. Howlett

\begin{abstract}
In this paper we prove that if $V$ is a vector space over a field of positive characteristic $p \neq 5$ then any regular subgroup $A$ of exponent 5 of $G L(V)$ is cyclic. As a consequence a conjecture of Gupta and Mazurov is proved to be true.
\end{abstract}

2000 Mathematics subject classification: primary 20E25, $20 \mathrm{~F} 50$.

\section{Introduction}

A group $G$ is called periodic if any element of $G$ has finite order and of finite exponent $e$ if, for any $g \in G$, we have $g^{e}=1$. Obviously any group of finite exponent is periodic, but the contrary is not true in general. We also recall that a group $G$ is called locally finite if each finite subset of $G$ is contained in a finite subgroup of $G$.

A well-known conjecture of Burnside says that a finitely generated group of finite exponent $e$ is necessarily finite (or, equivalently, that any group of finite exponent is locally finite).

This conjecture has been proved only for $e=2$ (in this case the group is abelian), for $e=3$ (Levi and van der Waerden [4], see also [8, 14.2.2]), for $e=4$ (Sanov [9], see also [8, 14.2.3]) and for $e=6$ (Hall [3]), while nothing is known for the case $e=5$. In some classes of groups Burnside's conjecture is true; for example, Burnside proved that if $F$ is a field of characteristic 0 , then any subgroup of finite exponent of $G L(n, F)$ is finite. However Burnside's conjecture is not true in general, as Novikov and Adjan proved in a series of papers of great length. Successively Adjan constructed infinite groups of exponent $e$ with a finite numbers of generators for any odd exponent $e \geq 665$ (see [1]).

(C) 2004 Australian Mathematical Society 1446-7887/04 $\$$ A2.00+0.00 
It is therefore quite natural to ask if, given a natural number $e$ and a vector space $V$ over a field $F$ of characteristic finite and coprime with $e$, there exists an infinite subgroup $A$ of $G L(V)$ of exponent $e$ that is regular (that is, with the property that $\alpha(v) \neq v$ for any $v \neq 0$ and any $\alpha \in A, \alpha \neq 1$ ). If $e$ is a prime number, it can be conjectured that $A$ is necessarily cyclic. This conjecture is certainly true if the dimension of $V$ over $F$ is finite (this fact was proved by Burnside; see $[8,10.5 .6]$ ).

In this paper, we consider the case $e=5$ and prove

THEOREM 1.1. If $V$ is a vector space over a field of positive characteristic $p \neq 5$ then any regular subgroup $A$ of exponent 5 of $G L(V)$ is cyclic.

We observe that the action of $A$ is regular over $V$ if and only if any non-identity element of $A$ has minimal polynomial that divide $x^{4}+x^{3}+x^{2}+x+1$. In grouptheoretic terms, this means that in the semidirect product of $V$ by $A$ there are not elements of order $5 p$.

\section{Notation and preliminary results}

We fix two distinct primes $p$ and $q$. Let $F$ be a field of characteristic $p, V$ a vector space over $F$ and $A$ a subgroup of the automorphism group of $V$ of exponent $q$ and such that for any $\alpha \in A, \alpha \neq 1$ we have $\operatorname{Fix}_{V}(\alpha)=\{0\}$. It is easy to verify that for any $\alpha \in A \backslash\{1\}$ and any $v \in V$ we have

$$
v+\alpha(v)+\alpha^{2}(v)+\cdots+\alpha^{q-1}(v)=0 .
$$

In the ring $\operatorname{End}_{F}(V)$ identity (1) can be written as follows

$$
1+\alpha+\alpha^{2}+\cdots+\alpha^{q-1}=0
$$

for any $\alpha \in A \backslash\{1\}$.

REMARK. For any pair of elements $\alpha, \beta \in A \backslash\{1\}$ with $\langle\alpha\rangle \cap\langle\beta\rangle=\{1\}$ we have $[\alpha, \beta] \neq 1$.

If $\alpha, \beta \in A \backslash\{1\}$ with $\langle\alpha\rangle \cap\langle\beta\rangle=\{1\}$ commute, then $\alpha \beta^{i}(i=0,1, \ldots, q-1)$ are all non identity elements of $A$. If we write the fundamental relation (2) for these elements, we get $1+\alpha \beta^{i}+\cdots+\left(\alpha \beta^{i}\right)^{q-1}=0$ for $i=0,1, \ldots, q-1$. Summing term by term and using the fact $[\alpha, \beta]=1$ we get

$$
q+\alpha\left(1+\beta+\cdots+\beta^{q-1}\right)+\cdots+\alpha^{q-1}\left(1+\beta+\cdots+\beta^{q-1}\right)=0
$$

but, by (2), $1+\beta+\cdots+\beta^{q-1}=0$, and therefore $q=0$ while $p \neq q$. This contradiction proves the statement. 
The preceding remark shows that any finite subgroup of $A$ must have order $q$. We observe that infinite groups in which any proper (non trivial) subgroup has order $q$ have been constructed by Ol'šanskiı ([7]). Groups of this type are called Tarski monsters.

Before proving Theorem 1.1, we want to expose the ideas behind the proof. We suppose for a moment that $q=3$ (and not knowing the theorem of Levi and van der Warden [4]); then we can write (2) as

$$
1+\alpha+\alpha^{-1}=0 \text { for all } \alpha \in A \backslash\{1\} .
$$

If $A$ is not cyclic, there exist $\alpha, \beta \in A \backslash\{1\}$ with $\langle\alpha\rangle \cap\langle\beta\rangle=\{1\}$ and from (3) we get

$$
\left\{\begin{array}{r}
1+\alpha+\alpha^{-1}=0 \\
1+\alpha \beta+\beta^{-1} \alpha^{-1}=0 \\
1+\alpha \beta^{-1}+\beta \alpha^{-1}=0
\end{array}\right.
$$

summing each member we obtain

$$
3+\alpha\left(1+\beta+\beta^{-1}\right)+\left(1+\beta+\beta^{-1}\right) \alpha^{-1}=0
$$

but, from (3), $1+\beta+\beta^{-1}=0$. From this we get the contradiction $3=0$ while $p \neq 3$.

\section{Proof of Theorem $1.1(p=2)$}

We suppose $q=5$; to prove Theorem 1.1 we suppose that there exists a counterexample, that is, a vector space $V$ over a field $F$ of characteristic $p \neq 5$ and a non cyclic group $A$ of exponent 5 acting regularly on $V$.

We fix the following notation: the indices in the sums will always be from 0 to 4 and considered mod 5 . We shall often use the fundamental relation (2) in the form

$$
1+\alpha+\alpha^{2}+\alpha^{3}+\alpha^{-1}=0
$$

or in the form

$$
1+\alpha+\alpha^{2}+\alpha^{-2}+\alpha^{-1}=0 .
$$

We shall always denote by $\alpha$ and $\beta$ two non identity elements of $A$ with $\langle\alpha\rangle \cap\langle\beta\rangle=\{1\}$.

The proof is in various steps.

STEP 1. We have $\sum_{i, j} \beta^{i+j} \alpha \beta^{i+2 j} \alpha \beta^{i+j}=0$. 
ProOF. If we put $i+j=r$ we obtain

$$
\sum_{i, j} \beta^{i+j} \alpha \beta^{i+2 j} \alpha \beta^{i+j}=\sum_{r}\left\{\beta^{r} \alpha \beta^{r}\left(\sum_{j} \beta^{j}\right) \alpha \beta^{r}\right\}
$$

and we conclude because $\sum_{j} \beta^{j}=0$.

We put $\bar{\sigma}=\sum_{i} \beta^{i} \alpha \beta^{i}$ and $\underline{\sigma}=\sum_{i} \beta^{i} \alpha^{-1} \beta^{i}$.

STEP 2. $\bar{\sigma}+\underline{\sigma}=0$.

Proof. If $i=0,1, \ldots 4$, by (4) we get

$$
1+\alpha \beta^{i}+\alpha \beta^{i} \alpha \beta^{i}+\alpha \beta^{i} \alpha \beta^{i} \alpha \beta^{i}+\beta^{-i} \alpha^{-1}=0
$$

summing the five preceding equalities and recalling that

$$
\alpha\left(\sum_{i} \beta^{i}\right)=0 \text { and }\left(\sum_{i} \beta^{-i}\right) \alpha^{-1}=0
$$

we get

$$
\alpha\left(\sum_{i} \beta^{i} \alpha \beta^{i}\right)+\alpha\left(\sum_{i} \beta^{i} \alpha \beta^{i} \alpha \beta^{i}\right)=-5
$$

and

$$
\sum_{i} \beta^{i} \alpha \beta^{i}+\sum_{i} \beta^{i} \alpha \beta^{i} \alpha \beta^{i}=-5 \alpha^{-1}
$$

The sum $\bar{\sigma}=\sum_{i} \beta^{i} \alpha \beta^{i}$ is invariant with respect to the substitutions $\alpha \leadsto \beta^{j} \alpha \beta^{j}$ with $j=0,1, \ldots, 4$. If we make these substitutions in (7) and we take a sum, we get

$$
5 \sum_{i} \beta^{i} \alpha \beta^{i}+\sum_{i, j} \beta^{i+j} \alpha \beta^{i+2 j} \alpha \beta^{i+j}=-5 \sum_{j} \beta^{-j} \alpha^{-1} \beta^{-j} .
$$

By Step 1 we have $\sum_{i, j} \beta^{i+j} \alpha \beta^{i+2 j} \alpha \beta^{i+j}=0$ and since char $F=p \neq 5$ we obtain the relation we wanted.

STEP 3. $\alpha \bar{\sigma}+\underline{\sigma} \alpha^{-1}=-5$.

PROOF. We observe that, since $A$ has exponent 5, the relation (6) can be written as $\alpha\left(\sum_{i} \beta^{i} \alpha \beta^{i}\right)+\left(\sum_{i} \beta^{-i} \alpha^{-1} \beta^{-i}\right) \alpha^{-1}=-5$.

STEP 4. $\bar{\sigma}^{2}+\underline{\sigma}^{2}=-25$. 
ProOF. We have observed before that $\bar{\sigma}$ and $\underline{\sigma}$ are invariant with respect to the substitutions $\alpha \leadsto \beta^{j} \alpha \beta^{j}$ with $j=0,1, \ldots, 4$. So we make these substitutions in $\alpha \bar{\sigma}+\underline{\sigma} \alpha^{-1}=-5$, we sum the five equalities and we get the desired result.

STEP 5. Theorem 1.1 is true if $p=2$.

Proof. Let $p=2$. By Step 2 we have $\bar{\sigma}=\underline{\sigma}$ and, recalling Step 4 we obtain the following contradiction $0=2 \bar{\sigma}^{2}=\bar{\sigma}^{2}+\underline{\sigma}^{2}=-25$.

\section{Proof of Theorem $1.1(p=3)$}

From now on, we suppose that $p=3$ and therefore the relations obtained in Steps $2-4$ have the form:

$$
\left\{\begin{aligned}
\bar{\sigma}+\underline{\sigma} & =0, \\
\alpha \bar{\sigma}+\underline{\sigma} \alpha^{-1} & =1, \\
\bar{\sigma}^{2}+\underline{\sigma}^{2} & =2 .
\end{aligned}\right.
$$

In particular, $\bar{\sigma}^{2}=\underline{\sigma}^{2}=1$.

STEP 6. We have

(a) $\alpha \bar{\sigma}=1+\bar{\sigma} \alpha^{-1}$

(b) $\alpha^{-1} \bar{\sigma}=\bar{\sigma} \alpha-1$.

PROOF. From $\bar{\sigma}=-\underline{\sigma}$ and from $\alpha \bar{\sigma}+\underline{\sigma} \alpha^{-1}=1$ we get (a).

Multiplying $\alpha \bar{\sigma}+g \alpha^{-1}=1$ on the left by $\alpha^{-1}$ and on the right by $\alpha$ we obtain $\alpha^{-1} \underline{\sigma}+\bar{\sigma} \alpha=1$ that gives (b).

STEP 7. If we put $\rho=\alpha+\alpha^{-1}$ and $\varphi=\alpha \bar{\sigma}$ we get

(a) $\rho \in G L(V)$ has order 8 and $\rho^{2}=1-\rho$;

(b) $\varphi \in G L(V)$ has order 8 and $\varphi^{2}=1+\varphi$;

(c) $[\rho, \varphi]=1$.

Proof. From the relations obtained in Step 6, we get

$$
\rho \bar{\sigma}=\left(\alpha+\alpha^{-1}\right) \bar{\sigma}=1+\bar{\sigma} \alpha^{-1}+\bar{\sigma} \alpha-1=\bar{\sigma}\left(\alpha+\alpha^{-1}\right)=\bar{\sigma} \rho
$$

and therefore $[\rho, \bar{\sigma}]=1$; since $[\rho, \alpha]=1$ we also have $[\rho, \varphi]=1$. Then

$$
\begin{aligned}
& \rho^{2}=\left(\alpha+\alpha^{-1}\right)^{2}=\alpha^{2}+\alpha^{-2}+2=-1-\alpha-\alpha^{-1}+2=1-\rho \quad \text { and } \\
& \rho^{4}=(1-\rho)^{2}=1-2 \rho+\rho^{2}=1-2 \rho+1-\rho=-1 .
\end{aligned}
$$


In particular, $\rho \in G L(V)$ and $\rho^{8}=1$. Moreover,

$$
\begin{aligned}
& \varphi^{2}=\alpha \bar{\sigma} \alpha \bar{\sigma}=\alpha\left(1+\alpha^{-1} \bar{\sigma}\right) \bar{\sigma}=1+\alpha \bar{\sigma}=1+\varphi \text { and } \\
& \varphi^{4}=(1+\varphi)^{2}=1+2 \varphi+\varphi^{2}=1+2 \varphi+1+\varphi=-1 .
\end{aligned}
$$

In particular, $\varphi \in G L(V)$ and $\varphi^{8}=1$.

STEP 8. The group $B=\left\langle\rho^{2}, \varphi^{2}\right\rangle \leq G L(V)$ is abelian and $|B| \leq 4$.

ProOF. By Step 7, $B$ is certainly abelian, moreover $\rho^{2}$ and $\varphi^{2}$ have order 4 and therefore, since $\rho^{4}=-1=\varphi^{4},|B| \leq 8$. We prove that $B$ has order (at most) 4 showing that $\rho^{2} \varphi^{-2}$, which has order 2 , acts fixed points freely over $V$ and it is therefore equal to -1 .

If we put $V_{0}=\operatorname{Fix}_{V}\left(\rho^{2} \varphi^{-2}\right)$ we have that $V_{0}$ is a $\langle\rho, \varphi\rangle$-invariant subspace of $V$ (because $\langle\rho, \varphi\rangle$ is abelian).

If, by contradiction, $V_{0} \neq\{0\}$ and using the same symbols for the restrictions of the automorphisms to $V_{0}$, from Step 7 we get $1-\rho=\rho^{2}=\varphi^{2}=1+\varphi$, that is, $\alpha \bar{\sigma}=\varphi=-\rho=-\alpha-\alpha^{-1}$. Using Step 6 (a) we get $1+\bar{\sigma} \alpha^{-1}=-\alpha-\alpha^{-1}$ and $\bar{\sigma}=-1-\alpha-\alpha^{2}$ and $1=\bar{\sigma}^{2}=1+\alpha+\alpha^{2}+\alpha^{4}+2 \alpha+2 \alpha^{2}+2 \alpha^{3}=1+2 \alpha+2 \alpha^{3}+\alpha^{4}$, that is, $\alpha^{4}=\alpha+\alpha^{3}$ and $\alpha^{2}=\alpha+\alpha^{-1}=\rho$ which gives the required contradiction: $1=\rho^{8}=\left(\alpha^{2}\right)^{8}=\alpha$.

STEP 9. Theorem 1.1 is true if $p=3$.

Proof. By Step 8 we have $|B| \leq 4$ and since $\rho^{4}=-1=\varphi^{4}$, this is possible only in two ways:

(I) $\rho^{2}=\varphi^{2}$ but this gives a contradiction, because in the proof of Step 8 we have seen that $\rho^{2} \varphi^{-2}$ acts fixed points freely on $V$.

(II) $\rho^{2}=-\varphi^{2}$ then, by Step 7, $1-\rho=-1-\varphi$ and $\varphi=1+\rho$. Then, recalling Step $6,1+\bar{\sigma} \alpha^{-1}=\alpha \bar{\sigma}=\varphi=1+\rho$ and $\bar{\sigma}=\rho \alpha=1+\alpha^{2}$; this implies $1=\bar{\sigma}^{2}=\left(1+\alpha^{2}\right)^{2}=1+2 \alpha^{2}+\alpha^{4}$ and $\alpha^{2}=1$ : a contradiction.

\section{Sketch of the proof of Theorem 1.1 for $p \geq 7$}

We remark that if char $F=p \geq 7$, we can obtain the same result in a way similar to the one used for $p=3$, but using arguments $a d$ hoc for any prime number $p$.

We can always find commuting elements $\rho$ and $\varphi$ (as defined in Step 7), satisfying $\rho^{2}+\rho-1=0$ and $\varphi^{2}+5 \varphi+2^{-1} \cdot 25=0$. The orders of these automorphisms are divisors of $p^{2}-1$ and depends on the prime $p$, as Table 1 shows, but we haven't been able to find a method of proof valid for any $p$.

It seems hard to prove the same conjecture for $A$ in the case in which $q=7$ (or greater), with the methods used in this paper. 
TABLE 1.

\begin{tabular}{|c|llllll|}
\hline$p$ & 3 & 7 & 11 & 13 & 17 & 19 \\
$|\rho|$ & 8 & 16 & 10 & 28 & 36 & 18 \\
$|\varphi|$ & 8 & 24 & 40 & 12 & 4 & 72 \\
\hline
\end{tabular}

\section{An application}

If $G$ is a periodic group, we denote by $\omega(G)$ the set of the orders of the elements of $G$. In [2] Gupta and Mazurov proved that if $\omega(G)$ is a proper subset of $\{1,2,3,4,5\}$, then either $G$ is locally finite or there exists a normal nilpotent 5 -subgroup $N$ of $G$ such that $G / N$ is a group of exponent 5 . The same authors have conjectured that if $N \neq\{1\}$ then $G$ is locally finite. This conjecture is equivalent to

CONJECTURE ([2]). Let $A$ be an automorphism group of an elementary abelian $\{2,3\}$-group $G$ such that every non-trivial element of $A$ fixes in $G$ only the trivial element. If $A$ is of exponent 5 then $A$ is cyclic.

The conjecture is true by Theorem 1.1; hence we have proved:

THEOREM 6.1. If $\omega(G) \subseteq\{1,2,3,4,5\}$ and $\omega(G) \neq\{1,5\}$ then the group $G$ is locally finite.

To establish Theorem 6.1, we need (in addition to the results of [2]) the following facts:

- The groups of exponent 4 are locally finite ([9]).

- If $\omega(G)=\{1,2,3,4,5\}$ then $G$ is locally finite ([5]).

- If $\omega(G)=\{1,2,3,5\}$ then $G \simeq A_{5}$ ([10]).

We recall that if $\omega(G)=\{1,2\}$ then $G$ is elementary abelian, if $\omega(G)=\{1,3\}$ then $G$ is nilpotent of class at most $3([4])$, and that the groups $G$ with $\omega(G)=\{1,2,3\}$ are described in [6].

\section{References}

[1] S. I. Adyan, 'Periodic groups of odd exponent', in: Proceedings of the Second International Conference on the Theory of Groups, Australian Nat. Univ., 1973, Lecture Notes in Math. 372 (Springer, Berlin, 1974) pp. 8-12.

[2] N. D. Gupta and V. D. Mazurov, 'On groups with small orders of elements', Bull. Austral. Math. Soc. 60 (1999), 197-205.

[3] M. Hall, 'Solution of the Burnside problem for exponent six', Illinois J. Math. 2 (1958), 764-786. 
[4] F. W. Levi and B. L. van der Waerden, 'Über eine besondere Klasse von Gruppen', Abh. Math. Sem. Univ. Hamburg 9 (1932), 154-158.

[5] V. D. Mazurov, 'Groups of exponent 60 with prescribed orders of elements', Algebra i Logika 39 (2000), 329-346; English translation: Algebra and Logic 39 (2000), 189-198.

[6] B. H. Neumann, 'Groups whose elements have bounded orders', J. London Math. Soc. 12 (1937), 195-198.

[7] A. Ju. Ol'šanskiI, 'An infinite group with subgroups of prime order', Izv. Akad. Nauk SSSR Ser. Mat. 44 (1980), 309-321.

[8] D. J. S. Robinson, A course in the theory of groups (Springer, Berlin, 1982).

[9] I. N. Sanov, 'Solution of Burnside's problem for exponent 4', Leningrad Univ. Ann. Math. Ser. 10 (1940), 166-170.

[10] A. K. Zhurtov and V. D. Mazurov, 'A recognition of simple groups $L_{2}\left(2^{m}\right)$ in the class of all groups', Sibirsk. Math. Zh. 40 (1999), 75-78; English translation: Siberian Math. J. 40 (1999), $62-64$.

Dipartimento di Informatica

Universitá di Ca' Foscari

Via Torino 155-30174 Venezia

Italy

e-mail: jabara@dsi.unive.it 